\title{
Rejeição de transplantes de córnea: tratamento tópico vs. pulsoterapia - resultados de 10 anos
}

\author{
Corneal allograft rejection: topical treatment vs. pulsed intravenous \\ methylprednisolone - ten years' result
}

\author{
Dácio CarvalhoCosta ${ }^{1}$ \\ Rosane Silvestre de Castro ${ }^{2}$ \\ Mariela Soares Ferraz de Camargo ${ }^{3}$ \\ Newton Kara-José ${ }^{4}$
}

Trabalho realizado no Hospital das Clínicas da Universidade Estadual de Campinas HC-UNICAMP - Campinas (SP) - Brasil.

Pós-graduando do curso de Ciências Médicas, área de concentração em Oftalmologia da Faculdade de Ciências Médicas da Universidade Estadual de Campinas UNICAMP - Campinas (SP) - Brasil.

Doutora em Oftalmologia pela Faculdade de Ciências Médicas da UNICAMP - Campinas (SP) - Brasil. Chefe do setor de Córnea e Doenças Externas do Hospital das Clínicas da UNICAMP.

Estagiária do Setor de Córnea e Doenças Externas do Hospital das Clínicas da UNICAMP - Campinas (SP) Brasil.

Professor Titular de Oftalmologia da Faculdade de Ciências Médicas da UNICAMP - Campinas (SP) Brasil.

Endereço para correspondência: Dácio Carvalho Costa. Rua Napoleão de Barros, 874 - Apto. 64 - São Paulo (SP) CEP 04024-002

E-mail:daciocosta@hotmail.com

Recebido para publicação em 27.11.2006

Última versão recebida em 19.09.2007

Aprovação em 25.09.2007

Nota Editorial: Depois de concluída a análise do artigo sob sigilo editorial e com a anuência da Dra. Núbia Cristina Freitas Maia sobre a divulgação de seu nome como revisora, agradecemos sua participação neste processo.

\section{RESUMO}

Objetivos: Avaliar a eficácia da associação de pulsoterapia com $500 \mathrm{mg}$ de metilprednisolona intravenosa ao acetato de prednisolona $1 \%$ tópico no tratamento do primeiro episódio de rejeição endotelial de transplantes de córnea. Métodos: Estudo caso-controle retrospectivo com 81 sujeitos que apresentaram o primeiro episódio de rejeição endotelial e submetidos à terapia nos primeiros quinze dias dos sintomas. Resultados: 67 sujeitos foram tratados com acetato de prednisolona $1 \%$ tópico de $1 \mathrm{em} 1$ hora e pulsoterapia com $500 \mathrm{mg}$ de metilprednisolona intravenosa no dia do diagnóstico e 14 sujeitos foram submetidos apenas ao tratamento com acetato de prednisolona $1 \%$ tópico formando o grupo controle. Dos 67 sujeitos submetidos a corticoterapia venosa e tópica, $41(61,19 \%)$ evoluíram satisfatoriamente e $26(38,8 \%)$ apresentaram falência endotelial. Dos 14 sujeitos submetidos apenas à corticoterapia tópica, $4(28,57 \%)$ evoluíram com enxerto transparente e os 10 restantes $(71,43 \%)$ com falência endotelial. $\mathrm{O}$ teste do qui-quadrado apontou maior taxa de sucesso $(\mathrm{p}<0,05)$ na reversão da rejeição endotelial do transplante de córnea no grupo submetido à pulsoterapia. Conclusões: $\mathrm{O}$ estudo sugere que o tratamento da rejeição endotelial de transplantes de córnea com pulsoterapia e corticóide tópico apresenta maior taxa de sucesso em relação ao uso isolado de corticóide tópico.

Descritores: Transplante de córnea/reabilitação; Rejeição de enxerto/terapia; Metilprednisolona/uso terapêutico; Metilprednisolona/administração \& dosagem; Complicações pós-operatórias; Corticosteróides/uso terapêutico

\section{INTRODUÇÃ̃O}

A ceratoplastia penetrante é o transplante de tecidos sólidos com a maior taxa de sucesso $^{(1)}$. A rejeição do enxerto doador é a complicação mais comum e freqüentemente leva à falência irreversível do transplante ${ }^{(2-3)}$. Na maior coorte estudada, a sobrevida do enxerto em 5 anos é de $73 \%{ }^{(3)}$. Em uma série brasileira, 17,69\% dos pacientes transplantados apresentaram rejeição em acompanhamento de cerca de $1 \mathrm{ano}^{(4)}$.

O uso de corticosteróides é a principal arma no tratamento da rejeição de transplantes de córnea desde os primeiros relatos ${ }^{(5-6)}$, porém a terapia com esteróides nem sempre é capaz de reverter os quadros de rejeição. A taxa de reversibilidade é influenciada por inúmeros fatores como o diagnóstico pré-operatório; a intensidade do edema de córnea quando do diagnóstico de rejeição; o tempo entre o início dos sintomas e o tratamento; a presença de fatores de risco no pré-operatório; o número de rejeições prévias; o tipo de tratamento adotado, entre outros ${ }^{(3-4,7-11)}$. Estima-se que 
entre $24-50 \%$ dos enxertos apresentem falência endotelial apesar do tratamento para rejeição ${ }^{(9,12-13)}$.

As vias de administração e os corticóides utilizados para o tratamento das rejeições de transplantes de córnea variam de acordo com a prática individual de cada oftalmologista. Estudos realizados nos Estados Unidos, Inglaterra e Austrália, baseados em questionários aplicados entre oftalmologistas que acompanham pacientes transplantados, mostram ampla variação na conduta diante de uma rejeição. O tratamento com corticóide tópico foi quase unânime e a droga de preferência da maioria dos entrevistados foi o acetato de prednisolona ${ }^{(14-16)}$.

Apenas em bases empíricas, alguns autores advogavam o uso de corticóides sistêmicos associados com corticóides tópicos para o tratamento de rejeições endoteliais graves. Dois trabalhos publicados no início da década de 90 influenciaram a conduta diante de rejeições endoteliais de transplante de córnea ${ }^{(17-18)}$. Em um estudo prospectivo ${ }^{(17)}$, alguns autores compararam o uso do pulso intravenoso de $500 \mathrm{mg}$ de metilprednisolona com a prednisona oral na dose $60-80 \mathrm{mg} / \mathrm{dia}$. Os pacientes transplantados que sofreram rejeição e foram tratados com pulsoterapia apresentaram maior sobrevida do enxerto. Por outro lado alguns autores em um estudo mais recente, concluíram não haver diferença estatisticamente significante entre o uso de pulsoterapia de metilprednisolona intravenosa associado ao tratamento tópico quando comparado ao tratamento tópico isolado ${ }^{(19)}$.

O presente estudo visa avaliar a eficácia da associação do pulso de $500 \mathrm{mg}$ de metilprednisolona intravenosa com acetato de prednisolona $1 \%$ tópico quando comparado ao uso único de acetato de prednisolona $1 \%$ tópico no tratamento da rejeição endotelial de transplantes de córnea.

\section{MÉTODOS}

Trata-se de estudo caso-controle, retrospectivo, realizado através de revisão de prontuários de 1.478 pacientes submetidos a ceratoplastia penetrante no Hospital das Clínicas da Universidade Estadual de Campinas (UNICAMP), durante o período de 1994 a 2004, e que apresentaram rejeição endotelial do transplante de córnea diagnosticada dentro dos seguintes critérios: edema estromal de córnea e presença de células no humor aquoso com (i) linha de rejeição endotelial, e/ou (ii) precipitados ceráticos no botão doador em um tecido previamente transparente.

Os critérios de inclusão foram: primeiro episódio de rejeição endotelial e tratamento até 15 dias do início dos sintomas. Foram excluídos os sujeitos com diagnóstico de herpes simples ocular, os que iniciaram o tratamento após o $16^{\circ}$ dia do início dos sintomas e os que já haviam apresentado rejeição endotelial previamente.

O protocolo seguido no Hospital das Clínicas da UNICAMP para o tratamento de rejeição endotelial de transplante de córnea consistia em: 1) uso de acetato de prednisolona $1 \%$ tópico de $1 \mathrm{em} 1$ hora durante uma semana a partir do dia do diagnóstico e 2) pulsoterapia com $500 \mathrm{mg}$ de metilprednisolona intravenosa no dia do diagnóstico de rejeição. Após 7 dias o paciente era reavaliado e, se houvesse melhora dos sinais e sintomas, procedia-se à redução lenta do corticóide tópico. Caso não houvesse melhora clínica, repetia-se o pulso de metilprednisolona e o paciente era novamente reavaliado em 7 dias, podendo haver ainda, caso fosse necessário, um terceiro pulso de metilprednisolona na terceira semana do diagnóstico de rejeição. Os sujeitos que seguiram este regime de tratamento formaram o grupo experimental.

Pacientes que apresentaram alguma contra-indicação clínica para pulso de corticóide intravenoso como diabetes e hipertensão arterial sistêmica descontrolada foram submetidos apenas a tratamento com corticóide tópico na mesma dose do grupo experimental, constituindo o grupo controle.

A reversão do episódio de rejeição de transplante de córnea foi determinada pelo exame à lâmpada de fenda e definida como retorno da córnea doadora ao estado de transparência e ausência de edema associado, com desaparecimento das células no humor aquoso.

$\mathrm{O}$ comitê de ética médica da universidade aprovou o estudo, de acordo com os termos da Declaração de Helsinque.

Os dois grupos foram comparados em relação à reversão do quadro de rejeição. Os dados obtidos foram submetidos à análise estatística através do software Minitab v13.

\section{RESULTADOS}

A revisão dos prontuários dos 1.478 pacientes submetidos a transplante de córnea de 1994 a 2004 no Serviço de Oftalmologia do Hospital das Clínicas da UNICAMP encontrou 81 indivíduos que atendiam os critérios de inclusão e exclusão do estudo. Deste total, 67 indivíduos foram submetidos a pulsoterapia com metilprednisolona intravenosa e corticóide tópico e 14 foram submetidos a tratamento tópico simples (grupo controle). Os dados epidemiológicos relativos a idade, gênero e diagnóstico pré-operatório estão apresentados nas tabelas 1 e 2 .

A média de pulsos no grupo submetido ao tratamento com $500 \mathrm{mg}$ de metilprednisolona foi de 1,97 pulso, com desvio padrão de 0,83 pulso. A tabela 3 mostra quantos sujeitos foram submetidos a 1, 2 ou 3 pulsos com o respectivo resultado do tratamento. Não houve diferença estatisticamente significante no resultado do tratamento da rejeição em relação ao número de pulsos realizados. Não foram observados efeitos colaterais sistêmicos do uso de metilprednisolona intravenosa no estudo.

Dos 67 sujeitos submetidos a pulsoterapia, $41(61,19 \%)$ evoluíram satisfatoriamente e $26(38,8 \%)$ apresentaram falência endotelial. Dos 14 sujeitos submetidos apenas a tratamento tópico, $4(28,57 \%)$ evoluíram com enxerto transparente e os 10 restantes $(71,43 \%)$ com falência endotelial. O teste do qui-quadrado foi aplicado para verificar se a relação entre a taxa de sucesso no tratamento da reversão de rejeição de 
transplantes de córnea com o uso do pulso de metilprednisolona intravenosa era superior à taxa de sucesso com o uso de tratamento tópico simples, obtendo-se resultado estatisticamente significante $(\mathrm{p}<0,05)$ (Tabela 4$)$. O odds ratio para o uso de pulso de metilprednisolona associado ao tratamento tópico comparado ao tratamento tópico único foi de 3,9423 (IC $95 \%=1,1189 ; 13,8895)$.

\section{DISCUSSÃO}

O tratamento da rejeição endotelial ainda é um dos maiores desafios para o sucesso das ceratoplastias ${ }^{(20)}$. Estima-se que, apesar do tratamento da rejeição, 24 a $50 \%$ dos enxertos apresentem falência ${ }^{(9,12-13)}$.

Pulsoterapia com corticosteróides tem sido utilizada em muitas áreas da medicina como reumatologia ${ }^{(21)}$, neurologia ${ }^{(22)}$ e hematologia ${ }^{(23)}$. Porém, o uso em oftalmologia é restrito apenas a doenças inflamatórias graves não responsivas ao tratamento tópico.

Em 1983, já havia sido preconizado o uso de $125 \mathrm{mg}$ de metilprednisolona para o tratamento de rejeições de transplante de córnea em bases empíricas ${ }^{(24)}$. Hill et al. ${ }^{(17-18)}$, referiram aumento do sucesso na terapia da rejeição dos transplantes de córnea com o uso de metilprednisolona $500 \mathrm{mg}$ em forma de pulso intravenoso em estudos prospectivos, porém o assunto ainda é controverso, principalmente por questões de segurança e mesmo, de eficácia ${ }^{(19,25-26)}$.

\begin{tabular}{|c|c|c|c|}
\hline Variável & $\begin{array}{l}\text { Grupo con- } \\
\text { trole }(n=14)\end{array}$ & $\begin{array}{l}\text { Grupo experi- } \\
\text { mental }(n=67)\end{array}$ & Significância \\
\hline $\begin{array}{l}\text { Gênero } \\
\text { (Masc:Fem) }\end{array}$ & $9: 5$ & $36: 31$ & $\mathrm{p}=0,522^{*}$ \\
\hline Idade (anos) & $38,4 \pm 21,9$ & $45,2 \pm 20,63$ & $\mathrm{p}=0,245^{\star \star}$ \\
\hline
\end{tabular}

\begin{tabular}{|c|c|c|}
\hline Causas & $\begin{array}{l}\text { Grupo controle } \\
(n=14)\end{array}$ & $\begin{array}{c}\text { Grupo experimental } \\
\qquad(n=67)\end{array}$ \\
\hline Ceratocone & $1(7,1 \%)$ & $26(38,8 \%)$ \\
\hline Distrofia de Fuchs & 0 & $2(3,0 \%)$ \\
\hline Desc Cx de catarata & $1(7,1 \%)$ & $5(7,5 \%)$ \\
\hline Úlcera perfurada & $10(71,4 \%)$ & $19(28,4 \%)$ \\
\hline Leucoma pós-trauma & $1(7,1 \%)$ & $1(1,5 \%)$ \\
\hline $\begin{array}{l}\text { Leucoma pós-úlcera } \\
\text { bacteriana }\end{array}$ & 0 & $3(4,5 \%)$ \\
\hline Anormal. congênitas & 0 & $2(3,0 \%)$ \\
\hline Falência de Tx prévio & $1(7,1 \%)$ & $6(9,0 \%)$ \\
\hline Tracoma & 0 & $3(4,5 \%)$ \\
\hline Total & $14(100 \%)$ & $67(100 \%)$ \\
\hline
\end{tabular}

No presente estudo, a taxa geral de sucesso para reversão de transplante de córnea foi de $55 \%$, o que situa a população estudada dentro de valores encontrados na literatura $^{(9,12-13)}$. Porém, a taxa de sucesso foi de $28,6 \%$ para o grupo tratado apenas com corticóide tópico e 61,2\% para o grupo tratado com corticóide tópico associado a pulsoterapia (Tabela 4). Este resultado foi estatisticamente superior $(\mathrm{p}<0,05)$ e o grupo tratado com a pulsoterapia apresentou cerca de 4 vezes mais chance de recuperação da rejeição $(\mathrm{OR}=3,9423$; IC 95\% = 1,1189; 13,8895).

A justificativa para o uso de pulso de metilprednisolona e seu maior sucesso no tratamento das rejeições endoteliais de transplantes de córnea repousa no fato de que a rejeição é uma reação sistêmica imunológica. O processo que desencadeia a rejeição do sistema imunológico ao tecido doador acontece nos linfonodos, fora do alcance dos esteróides tópicos. Corticosteróides sistêmicos acima de um certo limiar são capazes de anular um sistema imunológico ativado para a rejeição. Um pico sérico acima de 2,5 a $10 \mu \mathrm{g} / \mathrm{mL}$ de metilprednisolona, que é atingido pelo pulso de $500 \mathrm{mg}$ da droga, é capaz de induzir a apoptose dos linfócitos $\mathrm{T}$ periféricos contribuindo para a inativação do processo de rejeição ${ }^{(27)}$. A ação da pulsoterapia com metilprednisolona combina então um grande efeito antiinflamatório com a remoção dos linfócitos $\mathrm{T}$ circulantes.

O sucesso da reversão de rejeições do transplante de córnea dos sujeitos tratados apenas com acetato de prednisolona $1 \%$ tópico no presente estudo ficou abaixo do que foi encontrado por outros autores, especialmente Hudde et al., que em seu trabalho apresentaram $85 \%$ de sucesso com a terapia

\begin{tabular}{|c|c|c|c|}
\hline Número de pulsos & Fracasso & Sucesso & Total \\
\hline 1 & $\begin{array}{c}10 \\
41,7 \%\end{array}$ & $\begin{array}{c}14 \\
58,3 \%\end{array}$ & $\begin{array}{c}24 \\
100 \%\end{array}$ \\
\hline 2 & $\begin{array}{c}9 \\
42,9 \%\end{array}$ & $\begin{array}{c}12 \\
57,1 \%\end{array}$ & $\begin{array}{c}21 \\
100 \%\end{array}$ \\
\hline 3 & $\begin{array}{c}7 \\
31,8 \%\end{array}$ & $\begin{array}{c}15 \\
68,2 \%\end{array}$ & $\begin{array}{c}22 \\
100 \%\end{array}$ \\
\hline Total & 26 & 41 & 67 \\
\hline
\end{tabular}

\begin{tabular}{|c|c|c|c|}
\hline Tipo de tratamento & Fracasso & Sucesso & Total \\
\hline $\begin{array}{l}\text { Tratamento tópico } \\
\text { simples }\end{array}$ & $\begin{array}{c}10 \\
71,43 \%\end{array}$ & $\begin{array}{c}4 \\
28,57 \%\end{array}$ & $\begin{array}{c}14 \\
100 \%\end{array}$ \\
\hline $\begin{array}{l}\text { Pulso de } 500 \text { mg de } \\
\text { metilprednisolona + } \\
\text { tratamento tópico }\end{array}$ & $\begin{array}{c}26 \\
38,81 \%\end{array}$ & $\begin{array}{c}41 \\
61,19 \%\end{array}$ & $\begin{array}{c}67 \\
100 \%\end{array}$ \\
\hline Total & $\begin{array}{c}36 \\
44,44 \%\end{array}$ & $\begin{array}{c}45 \\
55,56 \%\end{array}$ & $\begin{array}{c}81 \\
100 \%\end{array}$ \\
\hline \multicolumn{4}{|c|}{ Qui-quadrado $=4,991, p=0,025, \mathrm{GL}=1$} \\
\hline
\end{tabular}


tópica isolada. Os autores atribuem esse alto índice de sucesso ao fato de que mesmo sendo apenas tópico, o tratamento destes pacientes foi realizado sob regime de internação, garantido que os mesmos recebessem o colírio de 1 em 1 hora até melhora clínica da rejeição ${ }^{(19)}$.

Os pacientes do presente estudo foram tratados ambulatorialmente, e assim não se pôde garantir que todos tivessem aderência ao uso correto da medicação, o que pode ser responsável, em parte, por essa taxa de sucesso mais baixa. No grupo tratado apenas com acetato de prednisolona $1 \%$ tópico, uma outra variável talvez possa ter influenciado para que este grupo apresentasse uma menor taxa de sucesso no tratamento da rejeição do transplante de córnea: o percentual de pacientes com úlcera de córnea foi maior no grupo controle, chegando a $71,4 \%$ (10/14), do que no grupo tratado com pulso de $500 \mathrm{mg}$ de metilprednisolona, que atingiu apenas 28,4\% (19/67) de pacientes com úlceras de córnea. A distribuição global da população estudada, porém, não apresentou uma diferença estatisticamente significante $(\mathrm{p}=0,1079)$. Sabe-se que o aumento da inflamação ocular, característica dos pacientes que sofreram transplante a quente, leva a uma maior chance de rejeição e falência.

O presente estudo tem como limitações àquelas de estudos caso-controle (retrospectivo e não randomizado), possuindo o grupo controle uma amostra pequena $(n=14)$.

\section{CONCLUSÃO}

O presente estudo sugere que o tratamento da rejeição endotelial em pacientes submetidos a transplante de córnea com a associação de metilprednisolona $500 \mathrm{mg}$ intravenosa e acetato de prednisolona $1 \%$ tópico apresenta maiores taxas de sucesso em relação ao uso isolado de acetato de prednisolona $1 \%$ tópico.

\section{ABSTRACT}

Purpose: To evaluate the efficacy of intravenous $500 \mathrm{mg}$ methylprednisolone in addition to topical treatment with $1 \%$ prednisolone in the treatment of the first episode of corneal endothelial rejection in patients that were submitted to corneal allograft transplantation. Methods: Retrospective casecontrol study with 81 patients that presented the first episode of corneal endothelial rejection and were treated within the first 15 days of the onset of symptoms. Results: 67 patients were treated with $1 \%$ topical prednisolone acetate and pulsed intravenous methylprednisolone $500 \mathrm{mg}$ at the diagnosis of corneal allograft rejection. Fourteen patients were submitted to topical treatment only, thus forming the control group. Forty-one of 67 patients (61.2\%) that were submitted to pulsed steroid had good outcome and $26(38.8 \%)$ presented corneal graft failure while only 4 of 14 patients (28.57\%) that received only topical steroids evolved with clear grafts and the remai- ning 10 patients $(71.43 \%)$ with graft failure. Chi-square showed statistically significant association $(\mathrm{p}<0.05)$ to greater success with pulsed methylprednisolone. Conclusions: This study suggests that the use of $500 \mathrm{mg}$ intravenous methylprednisolone in addition to $1 \%$ topical prednisolone acetate for the treatment of endothelial corneal allograft rejection presents better outcomes in reverting corneal allograft rejection when compared to isolated use of $1 \%$ topical prednisolone acetate.

Keywords: Corneal transplantation/rehabilitation; Graft rejection/therapy; Methylprednisolone/therapeutic use; Methylprednisolone/administration \& dosage; Postoperative complications; Adrenal cortex hormones/therapeutic use

\section{REFERÊNCIAS}

1. Barron B. Penetrating keratoplasty. In: Kaufman H, Barron B, McDonald M, editors. The cornea. Boston: Butterworth-Heinemann; c1998. p.805-45.

2. Williams KA, Muehlberg SM, Lewis RF, Coster DJ. Long-term outcome in corneal allotransplantation. The Australian Corneal Graft Registry. Transplant Proc. 1997;29(1-2):983.

3. Williams KA, Esterman AJ, Bartlett C, Holland H, Hornsby NB, Coster DJ. How effective is penetrating corneal transplantation? Factors influencing longterm outcome in multivariate analysis. Transplantation. 2006;81(6):896-901.

4. Chalita MR, Diazgranados EB, Sato EH, Branco BC, Freitas D. Rejeição corneana pós transplante de córnea: análise de dados do Banco de Olhos do Hospital São Paulo - Escola Paulista de Medicina. Arq Bras Oftalmol. 2000; 63(1):55-8.

5. Feldman JD. Graft rejection. Arch Intern Med. 1969;123(6):713-8. Review.

6. Coster DJ, Williams KA. Immunosuppression for corneal transplantation and treatment of graft rejection. Transplant Proc. 198921(1 Pt 3):3125-6. Review.

7. Bertelmann E, Jaroszewski J, Pleyer U. Corneal allograft rejection: current understanding. 2. Clinical implications. Ophthalmologica. 2002;216(1):2-12.

8. Charlín ER, López MM. Transplante corneal: análisis retrospectivo de una serie nacional. Arch Chil Oftalmol. 2004;61(2):13-20.

9. Naacke HG, Borderie VM, Bourcier T, Touzeau O, Moldovan M, Laroche L. Outcome of Corneal transplantation rejection. Cornea. 2001;20(4):350-3.

10. Dua HS, Azuara-Blanco A. Corneal allograft rejection: risk factors, diagnosis, prevention, and treatment. Indian J Ophthalmol. 1999;47(1):3-9.Review.

11. Tham VM, Abbott RL. Corneal graft rejection: recent updates. Int Ophthalmol Clin. 2002;42(1):105-13.

12. Alldredge OC, Krachmer JH. Clinical types of corneal transplant rejection. Their manifestations, frequency, preoperative correlates, and treatment. Arch Ophthalmol. 1981;99(4):599-604.

13. Gibbs D, Batchelor JR, Werb A, Schlesinger W, Casey TA. The influence of tissue-type compatibility on the fate of full-thickness corneal grafts. Trans Ophthalmol Soc UK. 1974;94(1):101-26.

14. Barker NH, Henderson TR, Ross CA, Coster DJ, Williams KA. Current Australian practice in the prevention and management of corneal allograft rejection. Clin Experiment Ophthalmol. 2000;28(5):357-60.

15. Koay PY, Lee WH, Figueiredo FC. Opinions on risk factors and management of corneal graft rejection in the United Kingdom. Cornea. 2005;24(3):292-6.

16. Rinne JR, Stulting RD. Current practices in the prevention and treatment of corneal graft rejection. Cornea. 1992;11(4):326-8.

17. Hill JC, Maske R, Watson P. Corticosteroids in corneal graft rejection. Oral versus single pulse therapy. Ophthalmology. 1991;98(3):329-33.

18. Hill JC, Ivey A. Corticosteroids in corneal graft rejection: double versus single pulse therapy. Cornea. 1994;13(5):383-8.

19. Hudde T, Minassian DC, Larkin DF. Randomised controlled trial of corticosteroid regimens in endothelial corneal allograft rejection. Br J Ophthalmol. 1999;83(12):1348-52. Comment in: Br J Ophthalmol. 2000;84(9):1083.

20. Polack FM. Rejeição no transplante de córnea. In: Cvintal T, editor. Complicações do transplante de córnea. São Paulo: Santos; c2004. p.203-8.

21. Liebling MR, Leib E, McLaughlin K, Blocka K, Furst DE, Nyman K, et al. Pulse methylprednisolone in rheumatoid arthritis: a double-blind cross-over trial. Ann Intern Med. 1981;94(1):21-6. 
22. Bracken MB, Shepard MJ, Holford TR, Leo-Summers L, Aldrich EF, Fazl M, et al. Administration of methylprednisolone for 24 or 48 hours or tirilazad mesylate for 48 hours in the treatment of acute spinal cord injury. Results of the Third National Acute Spinal Cord Injury Randomized Controlled Trial. National Acute Spinal Cord Injury Study. JAMA. 1997;277(20):1597-604.

23. Menichelli A, Del Principe D, Rezza E. Intravenous pulse methylprednisolone in chronic idiopathic thrombocytopenia. Arch Dis Child. 1984:59(8):777-9.

24. Braude LS, Chandler JW. Corneal allograft rejection. The role of the major histocompatibility complex. Surv Ophthalmol. 1983;27(5):290-305.
25. Thiel MA, Ross CA, Coster DJ. Corneal allograft rejection: has the time come for intravenous pulsed methylprednisolone? A debate. Clin Experiment Ophthalmol. 2000;28(6):398-404.

26. Teichmann KD. Randomised controlled trial of corticosteroid regimens in endothelial corneal allograft rejection. Br J Ophthalmol. 2000; 84(9): 1083 .

27. Migita K, Eguchi K, Kawabe Y, Nakamura T, Shirabe S, Tsukada T, et al Apoptosis induction in human peripheral blood $\mathrm{T}$ lymphocytes by high-dose steroid therapy. Transplantation. 1997;63(4):583-7.

\section{VENHA CONHECER AS BELEZAS DE FLORIPA}
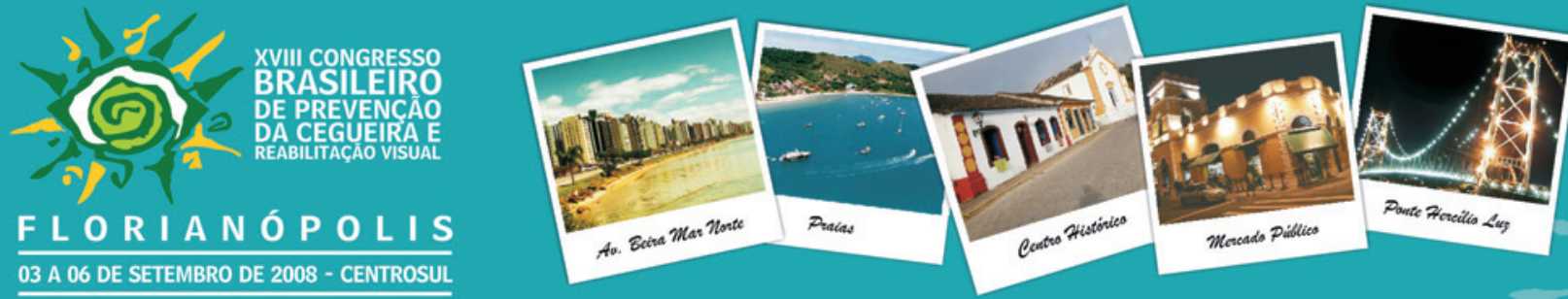

\section{UMA EXCELENTE VITRINE PARA OS SEUS CONHECIMENTOS}

Pela primeira vez a Comissão Científica está abrindo a oportunidade para que todos os médicos oftalmologistas possam inscrever-se a ministrar cursos no XVIII Congresso Brasileiro de Prevenção da Cegueira e Reabilitação Visual. Os temas, por sua relevância e interesse serão classificados pela Comissão Científica e farão parte da grade oficial de cursos do CBO 2008. Inscreva seu curso e propague seus conhecimentos.

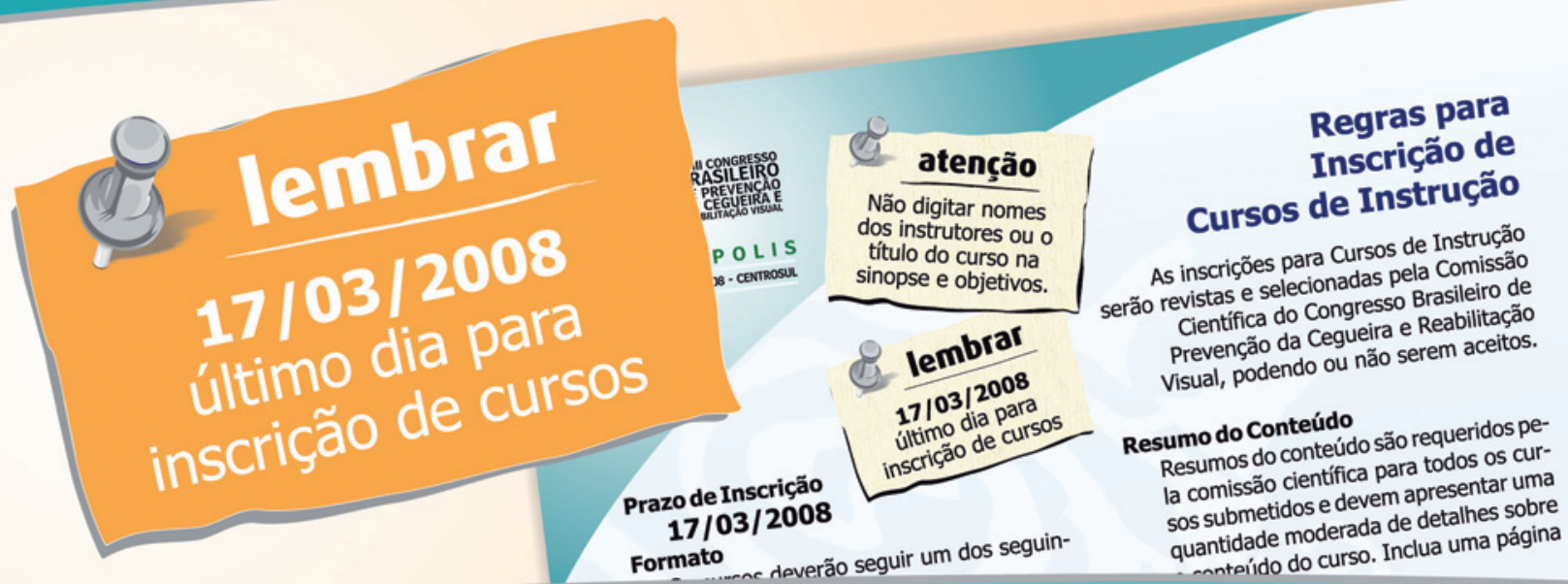

\section{Informações e inscrições: www.cbo2008.com.br}

REALIZAÇ̃̃o

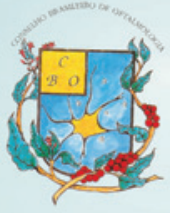

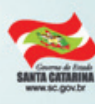

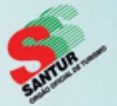

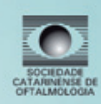

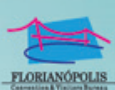

APOIO

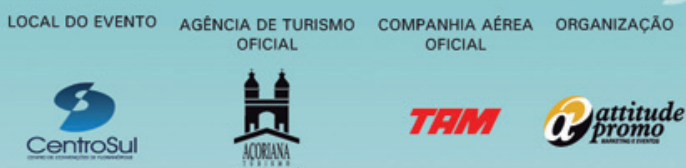

